\title{
Analog Digitized Data Logger with Wireless and Wired Communication Interface and RFID Features
}

\author{
Radek Kuchta, Radimir Vrba \\ Brno University of Technology, Department of Microelectronics \\ Udolni 53, Bmo, Czech Republic \\ \{kuchtar, vrbar\}@feec.vutbr.cz
}

\begin{abstract}
Knowledge of temperature-time curve for a certain time interval is needed in many scientific, medical and industrial applications. In some applications, however, the recorded temperature values in monitored time should be read wirelessly. Both requirements are defined especially for the transport of biological active substances. This set was applied in case study for continuous temperature data logging during a transport of chilled electrochemical TFT sensors with applied enzymes, highly sensitive to higher temperature limit exceeding.
\end{abstract}

Keywords: RFID, wireless communication, temperature measuring, data logging.

\section{Introduction}

A portable data logger with RFID features was designed for applications, where portability and wireless data transfer is inevitable. Communicating reader/writer can be mounted on the wall or can be portable, too. At the beginning it was designed for temperature measuring and monitoring, but the other analog sensors can be used, too.

Two main modes of operation for a tag during temperature data logging may be remotely chosen.

Mode 1 - standard storing of data in preprogrammed regular acquisition time intervals (100 milliseconds up to 2 hours) with number of samples limited only by a data EEPROM memory size.

Mode 2 - more memory size reducing method, where only breaking lower and upper temperature limits initiates storing the date and time stamp. However, the following date and time stamp is stored only when the temperature returns back into the temperature band between lower and upper temperature predefined limits. This mode 2 corresponds with a structure of data stored in data EEPROM memory. Enhanced mode can be set when the maximum or minimum temperature between breaking and returning points of a sampling temperature course is stored, too. This is a typical example for monitoring of foodstuff transport, where the time stamp and maximum temperature after breaking the limit are proofs to define the offender who damaged the transported goods. 
Wireless RFID systems generate and radiate electromagnetic waves. That's why they are legally classified as radio systems. The function of other radio services must under no circumstances be disrupted or impaired by the operation of RFID systems. For this reason, it is usually only possible to use frequency ranges that have been dedicated specifically for industrial, scientific or medical applications. These are the frequencies classified for worldwide as ISM (Industrial - Scientific - Medical), and they can also be used for RFID applications. The most important frequency ranges for RFID systems are therefore $135 \mathrm{kHz}, 27.125 \mathrm{MHz}, 40.68 \mathrm{MHz}, 433.92 \mathrm{MHz}, 869.0$ $\mathrm{MHz}, 915.0 \mathrm{MHz}, 2.45 \mathrm{GHz}, 5.8 \mathrm{GHz}$ and $24.125 \mathrm{GHz}$.

The range below $135 \mathrm{kHz}$ is heavily used by other radio services because it has not been reserved as an ISM frequency range. The propagation conditions in this long wave frequency range permit the radio services that occupy this range to reach wide areas at a low technical cost. In order to prevent collisions, the future Licensing Act for Inductive Radio Systems in Europe, $220 \mathrm{ZV} \mathrm{122,} \mathrm{will} \mathrm{define} \mathrm{a} \mathrm{protected} \mathrm{zone} \mathrm{of}$ between 70 and $119 \mathrm{kHz}$, which will no longer be allocated to RFID systems.

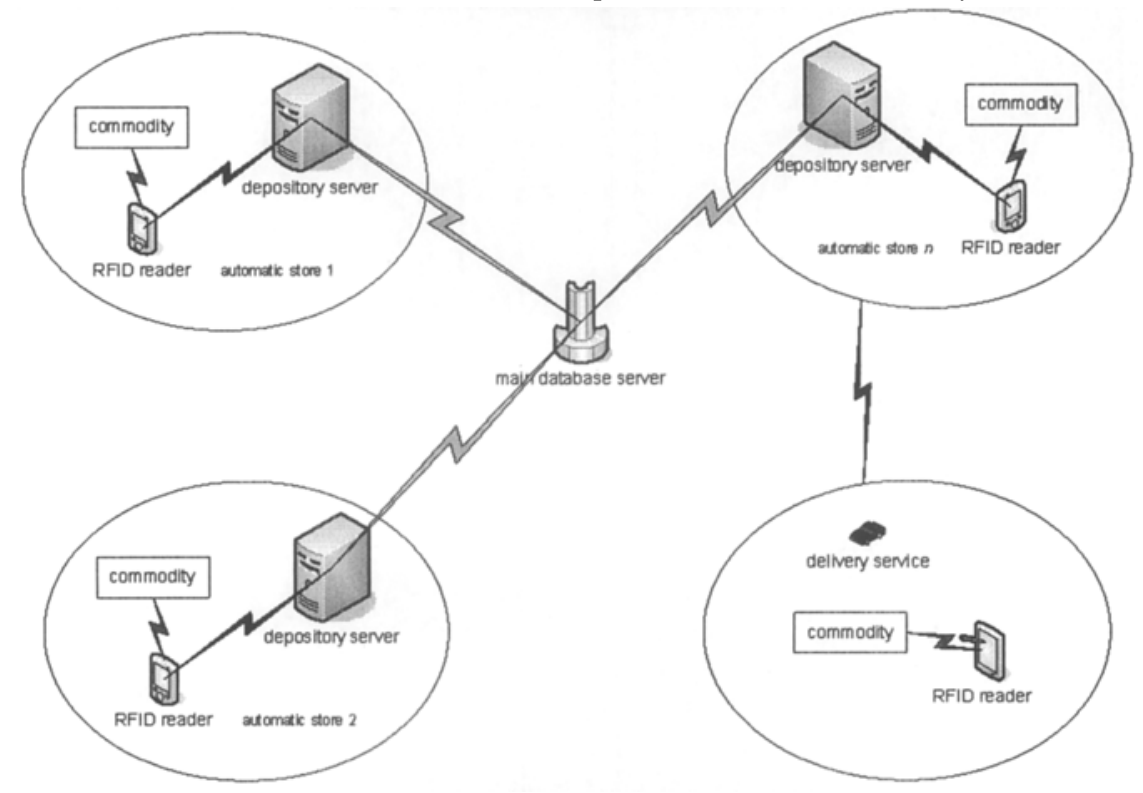

Fig. 1. Block diagram of temperature tag and reader/writer system

The main block diagram of designed tag and reader/writer system is shown in Fig. 1.

\section{Preferences for frequency range below $135 \mathrm{kHz}$}

This frequency range allows reaching large ranges with low cost transponders. High level of power is available to the transponder. The transponder has low power consumption due to its lower clock frequency and often sleeping a standby mode of operation. 
Miniaturized transponder formats can be achieved due to the use of ferrite coils in transponder. Low absorption rate or high penetration depth in non-metallic materials and water are available due to lower frequencies. Basic block diagram is shown in Fig. 2.

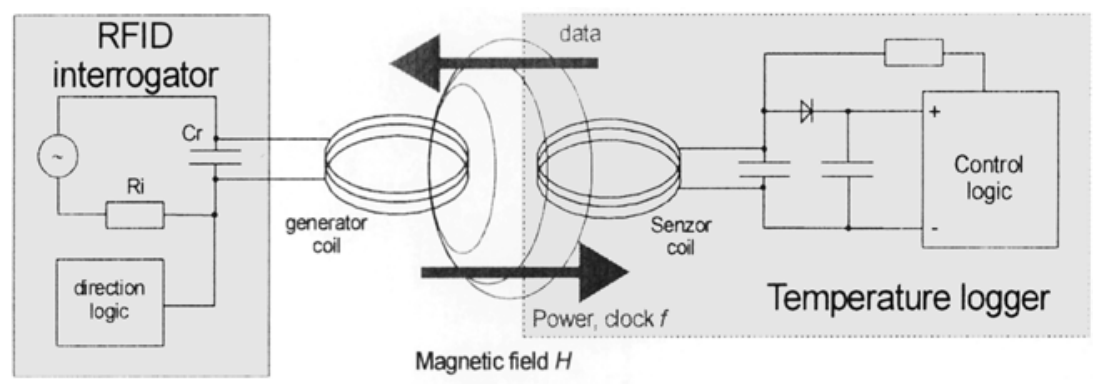

Fig. 2. Data transfer, and inductively coupled RFID interrogator and temperature data-logger block diagram

\section{Communication patterns}

An inductively coupled data logger comprises an electronic data-logging device and a large area coil that functions as an antenna. Inductively coupled logger is almost always operated in passive mode of data transmitting between an RFID tag and reader. This means that all the energy needed for the data transfer to the temperature logger has been provided by the reader. For this purpose, the reader's antenna coil generates a strong, high frequency electromagnetic field, which penetrates the crosssection of the coil area and the area around the coil.

\section{Main data-logger features}

Temperature is recorded using a temperature tag at user defined time intervals. The temperature tag can be programmed so that when the memory is full it either stops further recording or continues recording by overwriting the earliest of the previously recorded data.

Typical stored information contains: date and time stamp, temperature, temperature tag unique ID.

Recorded information can be transferred to a reader/writer and then to a $\mathrm{PC}$ or a PDA with wired or wireless connection to a reader/writer.

Temperature can be displayed graphically and the zoom functions allow focus on time periods where the temperature exceeds parameters.

The tag is a self-powered facility working like a wireless temperature sensor. The tag consists of a temperature probe and active part with active RFID technology. It is powered by an internal battery. The tag transmits an RF signal on demand at a pre-set time-interval. Tag life is estimated at several months depending on pre-set period of a 
transmission. The transmission interval can be configured via wireless connection by a reader/writer. The lifespan of the tag ends when the battery life is exhausted. Battery status can be inferred by interrogating the internal tag status value.

The lifespan of the tag can be increased by delayed turning on by the first communication attempt transmitted by the reader/writer.

\section{Temperature data logger tag}

This part of tag is used for temperature measuring, measured data storing and real time clock timing. Temperature is acquired in pre-set intervals. Tag can work in two basic modes as Data Logger and Out of Limit Values Logger.

- Data Logger - Temperature is measured in pre-set intervals. All data is stored into the internal memory. In this mode there is stored only a start time. Number of measurements depends on memory size. Data logging principle is shown in Fig. 3.

- Out of Limit Values Logger - If temperature is out of range, time stamp and temperature data are stored into the internal memory. Number of stored values depends on memory size. Out of Limit Values Logger principle is shown in Fig. 4.

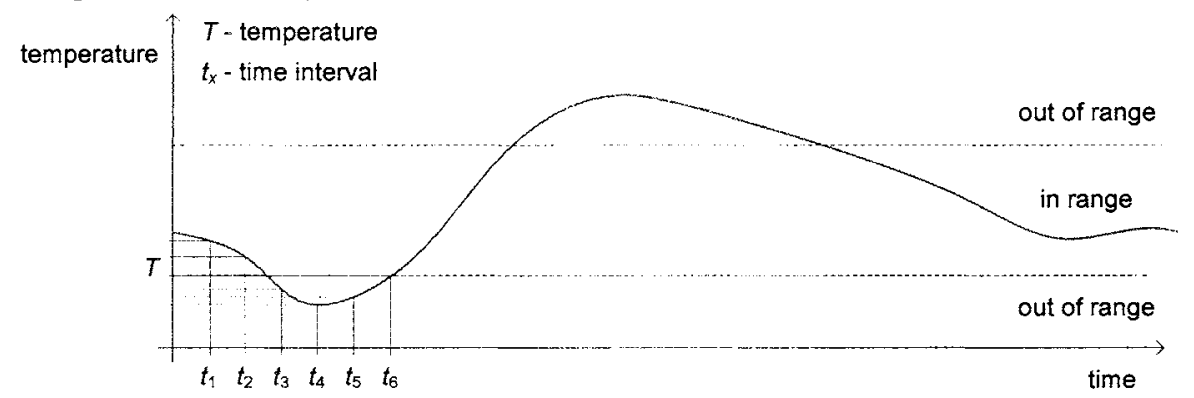

Fig. 3. Temperature data logging principle

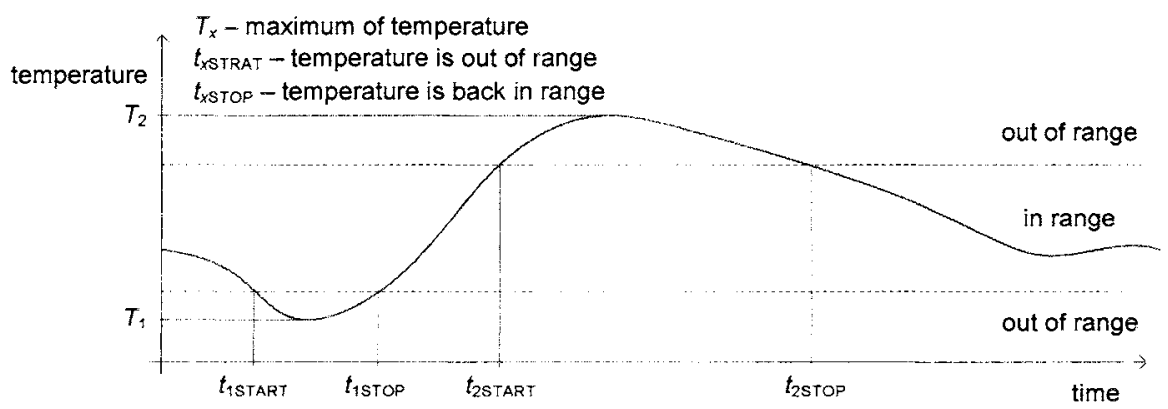

Fig. 4. Out of Limit Values Logger principle 


\section{Application areas}

The temperature tag is designed for usage in shipping containers, dairy industry, medical applications, fuel industry, refrigerated loads, agricultural industry, refrigeration monitoring and dangerous goods areas, anywhere temperature monitoring is required. On fig. 5 are both wireless and wired types of data logger with temperature sensor.
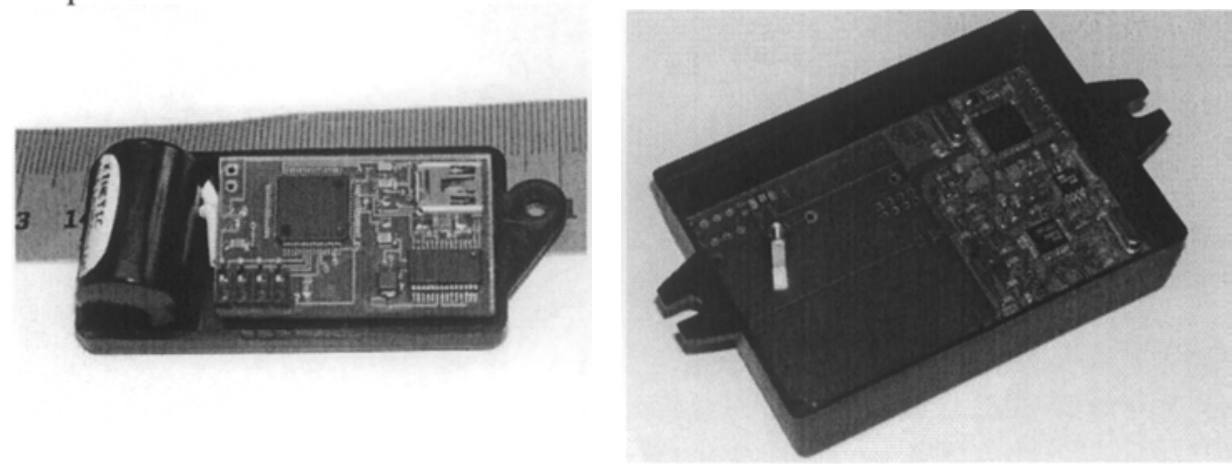

Fig. 5. Wired and wireless version of data logger

This set of applications increasingly means that temperature is a critical process and quality assurance factor for many industries.

The tag and reader/writer system is designed around a few simple ideas:

- Information is only good if it's accurate.

- Data is only useful if it's easily understood.

- Collected data is secure.

- A system is only good if it gets used.

- A system must be affordable.

- Simple setup and operation requiring minimal operator training.

\section{PC based communication software}

PC based communication software is used to setting up wired or wirelessly connected data logger. If wired connection is used, temperature data logger is connected directly via USB bus. In wireless connection RFID reader is necessary.

By this software tool is possible to set all selectable parameters. Setting up internal real time timer, temperature limits and work mode. One part is used for security setting.

When the connection is established all stored data is transferred to PC. In next step data can be saved to simple text file, plot to BMP picture or if the SQL server is available data is stored to the server database system. Main software window is on Fig. 6. 


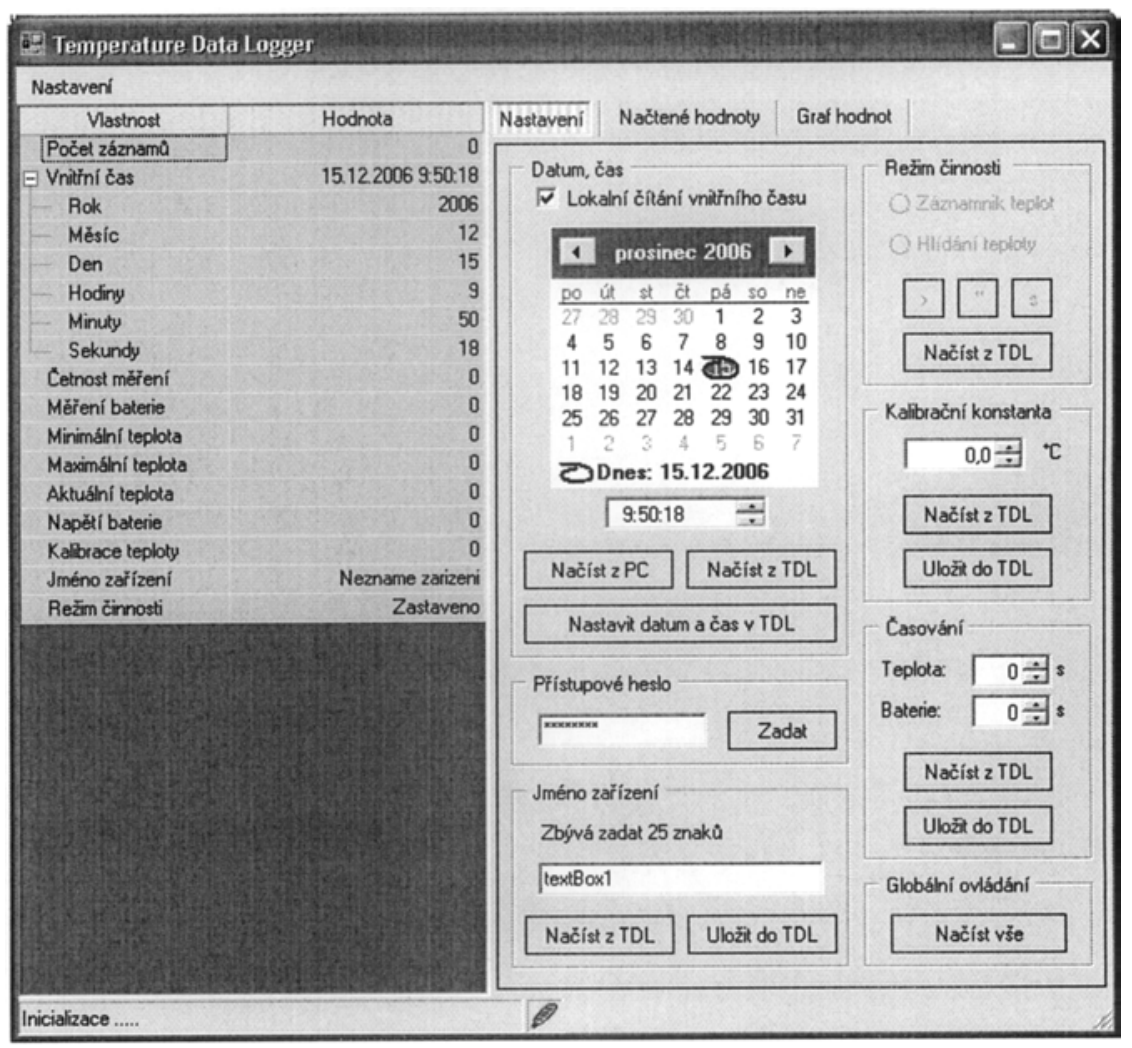

Fig. 6. PC based communication software

\section{Summary}

This contribution deals with a new method of portable wireless temperature data logging, which can be used for certified proof of history of temperature during monitored time interval. It fits to requirements defined by the transport of biological active substances. This set was applied for continuous temperature digital measuring and data logging during a transport of chilled electrochemical TFT sensors with applied enzymes. This type of sensors is highly sensitive and easily degradable when the temperature overcomes the maximum temperature limit. 
Acknowledgement. The research has been supported by the Czech Ministry of Education in the frame of MSM0021630503 MIKROSYN New Trends in Microelectronic Systems and Nanotechnologies, by the Czech Science Foundation as the project GACR 102/05/0869 ADC New Principles of Intergrated Low-Voltage and Low-Power AD Converters in Sub-Micron Technologies and by the Ministry of Industry and Commerce of the Czech Republic in 2A-1TP1/143 MEMS Research of New Mechatronic MEMS Structures Appropriate for Pressure Measurement.

\section{References}

1. Data sheets and manuals on www.microchip.com.

2. Data sheets and manuals on www.rfm.com.

3. M. Ilyas and 1. Mahgoub, (Eds.), Handbook of Sensor Networks: Compact Wireless and Wired Sensing Systems, CRC Press LLC, Boca Raton, FL, USA, 2004.

4. M. Sveda, P. Benes, R. Vrba and F. Zezulka, "Introduction to Industrial Sensor Networking," A book chapter in: M. Ilyas and I. Mahgoub, (Eds.), Handbook of Sensor Networks: Compact Wireless and Wired Sensing Systems, CRC Press LLC, Boca Raton, FL, USA, 2004.

5. D. Boling, "Programming Microsoft Windows CE .NET" Third edition, Handbook of Sensor Networks: Compact Wireless and Wired Sensing Systems, Microsoft Press, Redmond, Washington 98052-6399, USA, 2003, ISBN 978-0-7356-1884-8. 